\title{
Combination ipilimumab and radiosurgery for brain metastases: tumor, edema, and adverse radiation effects
}

\author{
Kevin Diao, BA, ${ }^{1,2}$ Shelly X. Bian, MD, ${ }^{2}$ David M. Routman, MD, ${ }^{2}$ Cheng Yu, PhD, ${ }^{2}$ Paul E. Kim, MD, ${ }^{3}$ \\ Naveed A. Wagle, MD, ${ }^{4}$ Michael K. Wong, MD, PhD, ${ }^{5}$ Gabriel Zada, MD, ${ }^{6}$ and Eric L. Chang, MD ${ }^{2}$ \\ ${ }^{1}$ Harvard Medical School, Boston, Massachusetts; and Departments of ${ }^{2}$ Radiation Oncology, ${ }^{3}$ Radiology, ${ }^{4}$ Clinical Neurology, \\ ${ }^{5}$ Medical Oncology, and ${ }^{6}$ Neurological Surgery, Keck School of Medicine of USC, Los Angeles, California
}

\begin{abstract}
OBJECTIVE Tumor and edema volume changes of brain metastases after stereotactic radiosurgery (SRS) and ipilimumab are not well described, and there is concern regarding the safety of combination treatment. The authors evaluated tumor, edema, and adverse radiation-induced changes after SRS with and without ipilimumab and identified associated risk factors.

METHODS This single-institution retrospective study included 72 patients with melanoma brain metastases treated consecutively with upfront SRS from 2006 to 2015. Concurrent ipilimumab was defined as ipilimumab treatment within 4 weeks of SRS. At baseline and during each follow-up, tumor and edema were measured in 3 orthogonal planes. The (length $\times$ width $\times$ height/2) formula was used to estimate tumor and edema volumes and was validated in the present study for estimation of edema volume. Tumor and edema volume changes from baseline were compared using the Kruskal-Wallis test. Local failure, lesion hemorrhage, and treatment-related imaging changes (TRICs) were analyzed with the Cox proportional hazards model.
\end{abstract}

RESULTS Of 310 analyzed lesions, 91 were not treated with ipilimumab, 59 were treated with concurrent ipilimumab, and 160 were treated with nonconcurrent ipilimumab. Of 106 randomly selected lesions with measurable peritumoral edema, the mean edema volume by manual contouring was $7.45 \mathrm{~cm}^{3}$ and the mean volume by (length $\times$ width $\times$ height)/2 formula estimation was $7.79 \mathrm{~cm}^{3}$ with $\mathrm{R}^{2}=0.99$ and slope of 1.08 on line of best fit. At 6 months after SRS, the ipilimumab groups had greater tumor $(p=0.001)$ and edema $(p=0.005)$ volume reduction than the control group. The concurrent ipilimumab group had the highest rate of lesion response and lowest rate of lesion progression $(p=0.002)$. Within the concurrent ipilimumab group, SRS dose $\geq 20$ Gy was associated with significantly greater median tumor volume reduction at 3 months $(p=$ 0.01 ) and 6 months $(p=0.02)$. The concurrent ipilimumab group also had the highest rate of lesion hemorrhage $(p=0.01)$. Any ipilimumab was associated with higher incidence of symptomatic TRICs $(p=0.005)$. The overall incidence of pathologically confirmed radiation necrosis (RN) was $2 \%$. In multivariate analysis, tumor and edema response at 3 months were the strongest predictors of local failure (HR 0.131 and HR 0.125) and lesion hemorrhage (HR 0.225 and HR 0.262). Tumor and edema response at 1.5 months were the strongest predictors of TRICs (HR 0.144 and HR 0.297).

CONCLUSIONS The addition of ipilimumab improved tumor and edema volume reduction but was associated with a higher incidence of lesion hemorrhage and symptomatic TRICs. There may be a radiation dose-response relationship between SRS and ipilimumab when administered concurrently. Early tumor and edema response were excellent predictors of subsequent local failure, lesion hemorrhage, and TRICs. The incidence of pathologically proven RN was low, supporting the relative safety of ipilimumab in radiosurgery treatment.

https://thejns.org/doi/abs/10.3171/2017.7.JNS171286

KEY WORDS brain metastases; stereotactic radiosurgery; ipilimumab; radiation necrosis; hemorrhage; edema; oncology

$\mathrm{S}$ TEREOTACTIC radiosurgery (SRS), which delivers highly conformal radiation in a single fraction, has been increasingly used for the treatment of brain metastases. SRS has better local control rates and fewer neurocognitive side effects compared with whole-brain radiation therapy (WBRT). ${ }^{1,2}$ Ipilimumab, a monoclonal antibody that blocks cytotoxic T-lymphocyte antigen-4 (CTLA-4), is an immune-modulating agent that was demonstrated to improve overall survival in metastatic melanoma patients during 2 Phase III randomized controlled

ABBREVIATIONS FLAIR = fluid-attenuated inversion recovery; GPA = graded prognostic assessment; IQR = interquartile range; KPS = Karnofsky Performance Status; RN = radiation necrosis; $\mathrm{SRS}=$ stereotactic radiosurgery; TRIC = treatment-related imaging change; WBRT = whole-brain radiation therapy.

SUBMITTED May 24, 2017. ACCEPTED July 14, 2017.

INCLUDE WHEN CITING Published online January 5, 2018; DOI: 10.3171/2017.7.JNS171286. 
trials. ${ }^{7,19}$ In these studies, the presence of brain metastases was an exclusion criterion. However, multiple retrospective studies have now found that ipilimumab, when combined with SRS for treatment of brain metastases, produces further improvements in intracranial control and overall survival. ${ }^{8,9,22,25}$

The toxicity profile of combination SRS and ipilimumab is not well understood. Some studies have found no increased toxicity compared with SRS alone, whereas a recent study found that the use of immunotherapy may lead to an increased incidence of radiation necrosis (RN), or its corresponding imaging finding, treatment-related imaging changes (TRICs). ${ }^{3,13}$ Furthermore, although there is evidence that immunotherapy produces different tumor response patterns compared with conventional chemotherapy, the trajectory-which we define as volume changes from baseline- of brain metastases and peritumoral edema following treatment with combination SRS and immunotherapy has not been well described in the literature. ${ }^{27}$

In this study, we report tumor and edema trajectories on MRI sequences of melanoma brain metastases treated with SRS with or without ipilimumab. We describe the effect of ipilimumab, timing of ipilimumab administration relative to SRS, and early tumor and edema response of lesions on subsequent outcomes, including local failure, lesion hemorrhage, TRICs, and RN.

\section{Methods \\ Patient Population and Data Collection}

This retrospective cohort study was approved by the USC Health Sciences Campus institutional review board. We reviewed records of consecutive patients treated with upfront SRS for melanoma brain metastases at our institution from 2006 to 2015. In patients undergoing additional SRS procedures, newly treated lesions were included in the study. We excluded patients who did not have any follow-up MRI studies and individual lesions that were resection cavities.

\section{Radiation and Ipilimumab Delivery}

All patients were treated with single-fraction Gamma Knife radiosurgery. Gamma Knife (Elekta AB) Model $\mathrm{C}$ and Perfexion were used for patients treated during 2006-2008 and 2008-2015, respectively. A stereotactic head frame was affixed to the cranium of the patient under conscious sedation. Afterward, contrast-enhanced MRI of the brain was performed for treatment planning. Radiation treatment was performed the same day. Prescription doses were based on lesion size as outlined in RTOG 9005. ${ }^{21}$

Patients received intravenous ipilimumab at a dose of either $3 \mathrm{mg} / \mathrm{kg}$ or $10 \mathrm{mg} / \mathrm{kg}$ scheduled every 3 weeks for up to 4 cycles. Concurrent ipilimumab was defined as ipilimumab administered within \pm 4 weeks of SRS, whereas administration outside this time frame was considered nonconcurrent. Our cutoff was based on the long 14.7-day half-life of ipilimumab, prior studies examining timing of ipilimumab using similar definitions, and clustering of patients for feasibility of statistical analysis. ${ }^{5,8,18}$ Patients not receiving ipilimumab were included as a control group.

\section{Imaging Evaluation}

Our institutional practice was to follow patients with MRI every 2-3 months after SRS, consistent with National Comprehensive Cancer Network (NCCN) guidelines. ${ }^{15}$ The maximum diameter of each tumor and edema was measured in 3 orthogonal planes on postgadolinium T1-weighted and T2 fluid-attenuated inversion recovery (FLAIR) MRI on the day of SRS treatment and for each subsequent follow-up evaluation. Postgadolinium T1 sequences had a slice thickness of 1-2 mm, while T2 FLAIR sequences had a slice thickness of 3-5 mm. The volume of each tumor or edema was estimated using the following validated formula: volume $=($ length $\times$ width $\times$ height $) / 2 .{ }^{6,12,18,23}$ The formula was validated for estimation of edema volume in the present study. Each follow-up volume was compared with baseline volume, and lesions were categorized as progressive ( $>30 \%$ volume increase), responsive ( $>30 \%$ volume decrease), or stable. These cutoffs were chosen based on an estimated volume measurement error of $\pm 30 \%$.

Local failure was defined as an increase in volume of $>30 \%$ from baseline either without subsequent resolution or requiring surgical intervention. TRICs were defined as an increase in volume of $>30 \%$ from baseline with subsequent resolution not requiring intervention. $\mathrm{RN}$ was defined as pathologically confirmed RN. Lesion hemorrhage was defined as the development of new intrinsic hyperintensity or increase in the volume of preexisting intrinsic T1 hyperintensity on precontrast T1-weighted MR images following SRS.

\section{Statistical Analysis}

Baseline patient and lesion characteristics were compared with the Kruskal-Wallis 1-way ANOVA test, Pearson chi-square test, and Fisher exact test. All further analysis was performed on a per-lesion basis. Tumor and edema volume change from baseline were compared with the Kruskal-Wallis 1-way ANOVA test. Significant values $(\mathrm{p}<0.05)$ were entered into pairwise testing using the Wilcoxon rank-sum test with Bonferroni correction for multiple comparisons. Tumor response at time intervals following SRS was compared with the Pearson chi-square test and Fisher exact test. Local failure, TRICs, RN, and lesion hemorrhage were analyzed as time-dependent variables using the Kaplan-Meier method with time calculated from day of SRS treatment and censoring occurring at intervention or last imaging follow-up. The Cox proportional hazards model was used to analyze the relationship between risk factors and local failure, TRICs, and lesion hemorrhage. All risk factors were entered into univariate analysis and significant variables were included in multivariate analysis. All statistical calculations were performed using JMP Pro (version 13; SAS Institute).

\section{Results}

A total of 72 patients and 310 brain metastases were included in analysis. The patients' median age was 61 years (interquartile range [IQR] 50-70), 23 (32\%) of the patients were female, the median Karnofsky Performance Status (KPS) score was 90 (IQR 80-90), and the median number of brain metastases was 2 (IQR 1-4). Of the 310 brain 
metastases, 91 were not treated with ipilimumab, 59 were treated with concurrent ipilimumab, and 160 were treated with nonconcurrent ipilimumab; 175 were treated with ipilimumab at a dose of $3 \mathrm{mg} / \mathrm{kg}, 31$ at a dose of $10 \mathrm{mg} /$ $\mathrm{kg}$, and dose information was not available for the other 13. Brain metastases were treated with a median radiation dose of $20 \mathrm{~Gy}$ (range 12-22 Gy). The median imaging follow-up time was 6.85 months.

To validate the (length $\times$ width $\times$ height $) / 2$ formula for estimation of peritumoral edema volume, 106 lesions with measurable peritumoral edema on T2 FLAIR MRI sequence were randomly selected to be manually contoured using Velocity (Varian Medical Systems). The mean ede- ma volume by contouring was $7.45 \mathrm{~cm}^{3}$ and the mean volume by (length $\times$ width $\times$ height) $/ 2$ formula estimation was $7.79 \mathrm{~cm}^{3}$ with $\mathrm{R}^{2}=0.99$ and slope of 1.08 on line of best fit.

\section{Patient and Lesion Characteristics}

Patients in the control (no ipilimumab), concurrent ipilimumab, and nonconcurrent ipilimumab groups did not differ significantly in terms of age, sex, KPS score, graded prognostic assessment (GPA), number of brain metastases, prior WBRT, or SRS dose (Table 1). Tumors in the control group had significantly greater baseline median volume $\left(0.15 \mathrm{~cm}^{3}\right)$ compared with the concurrent ipilimumab $(0.11$ $\left.\mathrm{cm}^{3}\right)$ and nonconcurrent ipilimumab $\left(0.09 \mathrm{~cm}^{3}\right)$ groups $(\mathrm{p}$

TABLE 1. Baseline and treatment characteristics for 72 patients with 310 brain metastases

\begin{tabular}{|c|c|c|c|c|}
\hline Characteristic & No Ipilimumab & Concurrent Ipilimumab & Nonconcurrent Ipilimumab & p Value \\
\hline \multicolumn{5}{|l|}{ Patient variables } \\
\hline Age, yrs & & & & 0.48 \\
\hline Median & 62 & 62 & 59 & \\
\hline IQR & $53-70.5$ & $38.25-74.5$ & $49-66.5$ & \\
\hline Sex & & & & 0.45 \\
\hline Male & $22 / 29(76 \%)$ & $12 / 18(67 \%)$ & $15 / 25(60 \%)$ & \\
\hline Female & $7 / 29(24 \%)$ & $6 / 18(33 \%)$ & $10 / 25(40 \%)$ & \\
\hline KPS score & & & & 0.61 \\
\hline Median & 90 & 90 & 90 & \\
\hline IQR & $80-90$ & $80-92.5$ & $80-90$ & \\
\hline GPA & & & & 0.60 \\
\hline Median & 1.5 & 2 & 2 & \\
\hline IQR & $1-2.38$ & $1.38-2.63$ & $1-2.25$ & \\
\hline No. of brain metastases & & & & 0.70 \\
\hline Median & 2 & 2.5 & 2 & \\
\hline IQR & $1-4$ & $1-3.5$ & $1-4$ & \\
\hline Prior WBRT & $3(10 \%)$ & $1(6 \%)$ & $2(8 \%)$ & 0.84 \\
\hline \multicolumn{5}{|l|}{ Lesion variables } \\
\hline Tumor location & & & & 0.87 \\
\hline Cerebral cortex & $81 / 91(90 \%)$ & $56 / 59(94 \%)$ & $140 / 160(88 \%)$ & \\
\hline Basal ganglia & $2 / 91(2 \%)$ & $1 / 59(2 \%)$ & $5 / 160(3 \%)$ & \\
\hline Cerebellum & $5 / 91(6 \%)$ & $1 / 59(2 \%)$ & $1 / 1601(7 \%)$ & \\
\hline Brainstem & $2 / 91(2 \%)$ & $1 / 59(2 \%)$ & $4 / 160(2 \%)$ & \\
\hline Tumor vol, $\mathrm{cm}^{3}$ & & & & 0.02 \\
\hline Median & 0.147 & 0.105 & 0.090 & \\
\hline IQR & $0.063-0.816$ & $0.050-0.495$ & $0.040-0.399$ & \\
\hline Edema vol, $\mathrm{cm}^{3}$ & & & & 0.04 \\
\hline Median & 0.550 & 0.280 & 0.160 & \\
\hline IQR & $0.063-2.736$ & $0.063-1.596$ & $0.050-1.365$ & \\
\hline Edema index ${ }^{*}$ & & & & 0.27 \\
\hline Median & 1.67 & 1.96 & 1.31 & \\
\hline IQR & $1-5.76$ & $1-5.60$ & $1-3.93$ & \\
\hline SRS dose, Gy & & & & 0.08 \\
\hline Median & 20 & 20 & 20 & \\
\hline IQR & $20-20$ & $18-20$ & $18-20$ & \\
\hline
\end{tabular}

Of the 72 patients, 29 received no ipilimumab, 18 received ipilimumab within 4 weeks $( \pm$ ) of SRS (concurrent ipilimumab), and 25 received ipilimumab outside of this time frame (nonconcurrent ipilimumab). With respect to the 310 individual lesions, the distribution was 91,59 , and 160 , respectively.

* Edema index = tumor volume/edema volume ratio. 

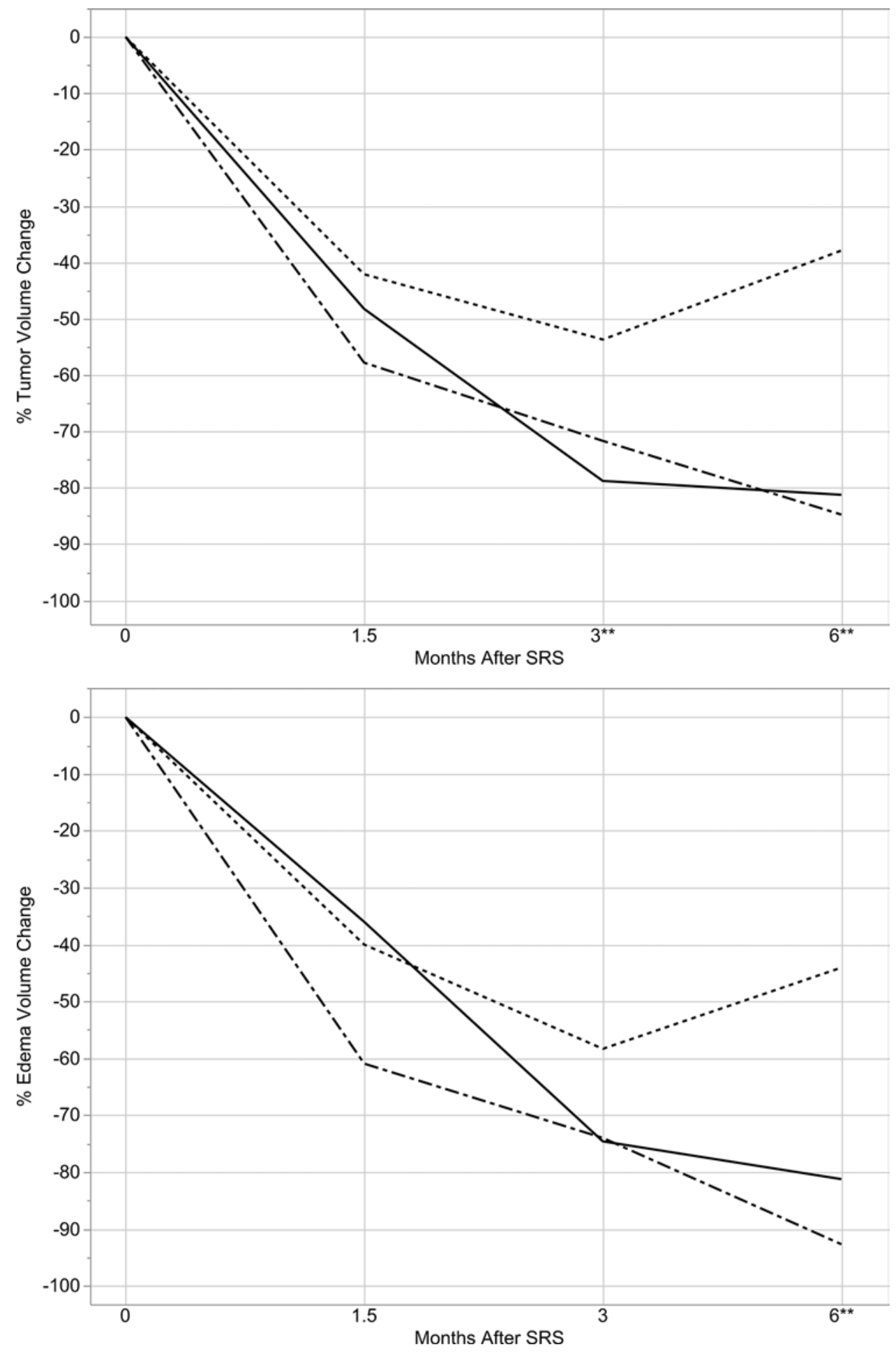

FIG. 1. Tumor (upper) and edema (lower) volume trajectories after SRS for no ipilimumab (dashed line), concurrent ipilimumab (dotted-dashed line), and nonconcurrent ipilimumab (solid line) at fixed time points. ${ }^{*}$ Statistically significant difference.

$=0.02$. Tumors in the control group also had larger baseline median edema volume $\left(0.55 \mathrm{~cm}^{3}\right)$ compared with the concurrent ipilimumab $\left(0.28 \mathrm{~cm}^{3}\right)$ and nonconcurrent ipilimumab $\left(0.16 \mathrm{~cm}^{3}\right)$ groups $(\mathrm{p}=0.04)$. The edema index, defined as the ratio of edema volume over tumor volume, did not differ significantly between the groups $(\mathrm{p}=0.27)$.

\section{Tumor and Edema Trajectory}

At 1.5 months after SRS, the difference in median tumor and edema volume change between the groups was not statistically significant $(\mathrm{p}=0.18$ and $\mathrm{p}=0.15$, respectively) (Fig. 1 and Table 2). At 3 months after SRS, the concurrent ipilimumab group had significantly greater tu- 
TABLE 2. Tumor and edema volume trajectories following SRS

\begin{tabular}{|c|c|c|c|c|c|}
\hline Time Since SRS \& Group & No. of Lesions & Tumor Vol Median \% Change & $\mathrm{p}$ Value & Edema Vol Median \% Change & p Value \\
\hline \multicolumn{6}{|l|}{$1.5 \mathrm{mos}$} \\
\hline No ipilimumab & 65 & -42 & 0.18 & -40 & 0.15 \\
\hline Concurrent ipilimumab & 55 & -48 & & -36 & \\
\hline Nonconcurrent ipilimumab & 135 & -58 & & -61 & \\
\hline \multicolumn{6}{|l|}{$3 \mathrm{mos}$} \\
\hline No ipilimumab & 41 & -54 & 0.05 & -58 & 0.15 \\
\hline Concurrent ipilimumab & 47 & -79 & & -75 & \\
\hline Nonconcurrent ipilimumab & 86 & -72 & & -74 & \\
\hline \multicolumn{6}{|l|}{$6 \mathrm{mos}$} \\
\hline No ipilimumab & 36 & -38 & 0.001 & -44 & 0.002 \\
\hline Concurrent ipilimumab & 43 & -81 & & -81 & \\
\hline Nonconcurrent ipilimumab & 88 & -85 & & -93 & \\
\hline
\end{tabular}

mor volume reduction compared with the control group ( $\mathrm{p}$ $=0.02$ ). At 6 months, both the concurrent and nonconcurrent ipilimumab groups had significantly greater tumor $(p$ $=0.001$ and $p=0.005$, respectively) and edema $(p=0.003$ and $\mathrm{p}=0.004$, respectively) volume reduction compared with the control group.

There was a significant difference in distributions of tumor response at 3 and 6 months following SRS. At both 3 and 6 months, the concurrent ipilimumab group had the highest rate of response ( $89 \%$ and $77 \%$, respectively) and lowest rate of progression ( $0 \%$ and $2 \%$, respectively) $(\mathrm{p}=$ 0.002 and $\mathrm{p}<0.001$, respectively) (Table 3).

Overall, dose $\geq 20$ Gy was not associated with significantly different median tumor volume change at 1.5 months $(-51 \%$ vs $-54 \%, \mathrm{p}=0.81), 3$ months $(-70 \%$ vs $-58 \%, \mathrm{p}=0.40)$, or 6 months $(-73 \%$ vs $-44 \%, \mathrm{p}=0.06)$. However, within the concurrent ipilimumab group, SRS dose $\geq 20$ Gy was associated with significantly greater median tumor volume reduction at 3 months $(-82 \%$ vs $-59 \%, \mathrm{p}=0.01)$ and 6 months $(-89 \%$ vs $-37.5 \%, \mathrm{p}=0.02)$. There were no significant associations within the nonconcurrent ipilimumab group or control group.

\section{Local Failure}

The overall incidence of local failure was $17 \%$. Concurrent ipilimumab treatment was associated with the lowest incidence of local failure, but the difference was not statistically significant $(10 \%, p=0.26)$ (Table 4). On univariate analysis, risk for local failure was decreased with concurrent ipilimumab (HR 0.34, 95\% CI 0.12-0.83, $\mathrm{p}=0.02$ ), tumor response at 1.5 months (HR $0.23,95 \%$ CI $0.11-0.46, p<0.001$ ), edema response at 1.5 months (HR $0.30,95 \%$ CI $0.15-0.58, \mathrm{p}<0.001$ ), tumor response at 3 months (HR $0.09,95 \%$ CI $0.03-0.19, \mathrm{p}<0.001$ ), and edema response at 3 months (HR 0.09, 95\% CI 0.03-0.20, $\mathrm{p}<0.001$ ), whereas risk for local failure was increased with tumor volume $>1 \mathrm{~cm}^{3}$ (HR 2.10,95\% CI 1.08-3.83, $\mathrm{p}=0.03$ ) (Table 5). In multivariate analysis, local failure remained significantly associated with concurrent ipilimumab (HR 0.36, 95\% CI 0.13-0.87, p = 0.02), tumor volume $>1 \mathrm{~cm}^{3}$ (HR 2.02, 95\% CI 1.03-3.70, p = 0.04), tumor response at 3 months (HR $0.13,95 \%$ CI $0.05-0.34$, p $<0.001$ ), and edema response at 3 months (HR $0.13,95 \%$ CI $0.04-0.31, \mathrm{p}<0.001)$.

\section{Lesion Hemorrhage}

The overall incidence of any lesion hemorrhage was $18 \%$ and symptomatic lesion hemorrhage was $5 \%$. Concurrent ipilimumab had higher incidence of any lesion hemorrhage $(p=0.01)$ but not symptomatic hemorrhage $(p=0.76)$. In univariate analysis of lesion hemorrhage, nonconcurrent ipilimumab was associated with lower risk of lesion hemorrhage (HR 0.48, 95\% CI 0.26-0.89, $\mathrm{p}=0.02)$ compared with concurrent ipilimumab. Cerebral hemisphere location was associated with higher risk of lesion hemorrhage (HR 6.87, 95\% CI 1.51-121.41, $\mathrm{p}=0.01$ ) compared with all

TABLE 3. Tumor response categorization at time points following SRS

\begin{tabular}{|c|c|c|c|c|c|c|c|c|c|c|}
\hline \multirow{2}{*}{$\begin{array}{c}\text { Time } \\
\text { Since SRS }\end{array}$} & \multicolumn{3}{|c|}{ No Ipilimumab } & \multicolumn{3}{|c|}{ Concurrent Ipilimumab } & \multicolumn{3}{|c|}{ Nonconcurrent Ipilimumab } & \multirow{2}{*}{$\begin{array}{c}p \\
\text { Value }\end{array}$} \\
\hline & Resp* & Stab† & Progf & Resp* & Stab† & Progf & Resp* & Stab† & Progf & \\
\hline $1.5 \mathrm{mos}$ & $45(69 \%)$ & $12(18 \%)$ & $8(12 \%)$ & $34(62 \%)$ & $14(25 \%)$ & $7(13 \%)$ & $94(70 \%)$ & $28(21 \%)$ & $13(10 \%)$ & 0.82 \\
\hline $3 \mathrm{mos}$ & $30(73 \%)$ & $4(10 \%)$ & $7(17 \%)$ & $42(89 \%)$ & $5(11 \%)$ & $0(0 \%)$ & $54(63 \%)$ & $15(17 \%)$ & $17(20 \%)$ & 0.002 \\
\hline $6 \mathrm{mos}$ & $20(56 \%)$ & $2(5 \%)$ & $14(39 \%)$ & $33(77 \%)$ & $9(21 \%)$ & $1(2 \%)$ & $59(67 \%)$ & $7(8 \%)$ & $22(25 \%)$ & $<0.001$ \\
\hline
\end{tabular}

Prog = progression; resp = response stab = stable.

Values are shown as the number of lesions (\%) unless otherwise indicated.

* $>30 \%$ tumor volume reduction.

$\dagger$ Neither $>30 \%$ tumor volume reduction nor increase.

$\ddagger>30 \%$ tumor volume increase. 
TABLE 4. Incidence of local failure, hemorrhage, TRICs, and RN

\begin{tabular}{lcccc}
\hline \multicolumn{1}{c}{ Outcome } & $\begin{array}{c}\text { No } \\
\text { Ipilimumab } \\
(\mathrm{n}=91)\end{array}$ & $\begin{array}{c}\text { Concurrent } \\
\text { Ipilimumab } \\
(\mathrm{n}=59)\end{array}$ & $\begin{array}{c}\text { Nonconcurrent } \\
\text { Ipilimumab } \\
(\mathrm{n}=160)\end{array}$ & $\begin{array}{c}\mathrm{p} \\
\text { Value }\end{array}$ \\
\hline Local failure & $17(19 \%)$ & $6(10 \%)$ & $30(19 \%)$ & 0.26 \\
\hline Lesion hemorrhage & $13(14 \%)$ & $19(32 \%)$ & $24(15 \%)$ & 0.01 \\
\hline Symptomatic & $5(5 \%)$ & $3(5 \%)$ & $6(4 \%)$ & 0.76 \\
\hline TRIC & $7(8 \%)$ & $8(14 \%)$ & $17(11 \%)$ & 0.50 \\
\hline Symptomatic & $0(0 \%)$ & $5(8 \%)$ & $9(6 \%)$ & 0.005 \\
\hline RN & $0(0 \%)$ & $2(3 \%)$ & $3(2 \%)$ & 0.22 \\
\hline
\end{tabular}

Values are shown as the number of lesions (\%) unless otherwise indicated.

other locations, which included brainstem, basal ganglia, and cerebellar lesions. Lesion hemorrhage was also associated with tumor response at 1.5 months (HR 0.56, 95\% CI $0.32-0.99, \mathrm{p}=0.04$ ), tumor response at 3 months (HR $0.29,95 \%$ CI $0.16-0.54, \mathrm{p}<0.001$ ), and edema response at 3 months (HR 0.30, 95\% CI 0.16-0.56, p < 0.001). In multivariate analysis, nonconcurrent ipilimumab (HR 0.40, $95 \%$ CI $0.21-0.76, p=0.01$ ), tumor response at 3 months (HR $0.23,95 \%$ CI $0.11-0.48, \mathrm{p}<0.001$ ), edema response at 3 months (HR 0.26 , 95\% CI $0.13-0.52$, p < 0.001), and cerebral hemisphere location (HR 6.31, 95\% CI 1.38-111.78, $\mathrm{p}=0.01$ ) remained associated with lesion hemorrhage.

\section{TRICs}

Overall, the incidence of TRICs was $10 \%$ and the incidence of symptomatic TRICs was 5\%. The median time to TRIC was 2.10 months for the control group, 1.93 months for the concurrent ipilimumab group, and 3.15 months for the nonconcurrent ipilimumab group $(p=0.99)$. There was no significant difference in the incidence of any TRICs between treatment groups, but patients receiving concurrent or nonconcurrent ipilimumab had significantly more symptomatic TRICs $(8 \%$ and $6 \%$, respectively, vs $0 \%$ for the no ipilimumab group, $\mathrm{p}=0.005$ ). The concurrent ipilimumab group had the largest TRICs (median 354\% volume increase from baseline), followed by the nonconcurrent ipilimumab (130\%) and no ipilimumab (63\%) groups. The difference was not statistically significant $(\mathrm{p}=0.42)$.

The median radiation dose to the lesions that developed into TRICs was 20 Gy (IQR 18-20 Gy) and for all other brain metastases was also 20 Gy (IQR 18-20 Gy) (p $=0.26$ ). Baseline median tumor size for lesions that progressed to TRICs was $0.16 \mathrm{~cm}^{3}$ (IQR $0.07-2.97 \mathrm{~cm}^{3}$ ) compared with $0.11 \mathrm{~cm}^{3}$ (IQR $0.05-1.99 \mathrm{~cm}^{3}$ ) for all others ( $\mathrm{p}$ $=0.23$ ). Lesions that developed into TRICs had a baseline median edema index of 1.99 (IQR 1-3), but this increased to 5.27 (IQR 1.32-9.73) at the maximum extent of TRIC $(\mathrm{p}<0.001)$. Among 25 brain metastases that progressed to TRICs in patients who received ipilimumab, 6 (24\%) were being treated with ipilimumab at the time of TRIC diagnosis.

In univariate analysis, TRICs were associated with tumor response at 1.5 months (HR $0.14,95 \%$ CI $0.06-0.31$, $\mathrm{p}<0.001$ ), edema response at 1.5 months (HR $0.22,95 \%$ CI $0.09-0.48, \mathrm{p}<0.001$ ), tumor response at 3 months (HR $0.30,95 \%$ CI $0.13-0.68, p=0.004)$, and edema response at
3 months (HR 0.25, 95\% CI 0.11-0.56, p = 0.002). In multivariate analysis, TRICs remained significantly associated with tumor response at 1.5 months (HR $0.14,95 \%$ CI $0.05-0.36, \mathrm{p}=0.005$ ) and edema response at 1.5 months (HR 0.30, 95\% CI 0.12-0.70, p < 0.001).

\section{Radiation Necrosis}

Overall, there was a low incidence of pathologically confirmed RN (2\%). Within the control group, $0(0 \%)$ of 91 lesions developed RN, whereas $2(3 \%)$ of 59 lesions treated with concurrent ipilimumab and 3 (2\%) of 160 lesions treated with nonconcurrent ipilimumab developed $\mathrm{RN}$. The difference between the groups was not statistically significant $(\mathrm{p}=0.22)$.

\section{Discussion}

The effect that the addition of ipilimumab to SRS has on the tumor and edema trajectories of brain metastases is not well understood. Furthermore, although there is concern that SRS and ipilimumab may place patients at increased risk for adverse radiation effects, there is no consensus on the safety of combination treatment.

\section{Tumor and Edema Trajectory}

We found that at 1.5-, 3-, and 6-month follow-up intervals, edema trajectory closely mirrored tumor trajectory, extending the findings of previous studies to the setting of combination SRS and ipilimumab. ${ }^{17}$ In our study, lesions receiving ipilimumab had greater tumor and edema volume reduction than those that did not receive ipilimumab by 6 months. Thus, despite concerns that the immunomodulatory effect of ipilimumab may produce a proinflammatory environment leading to increased peritumoral edema of brain metastases, we found that the addition of ipilimumab to SRS produced a trend toward improved edema control at 1.5 and 3 months, and significantly improved edema control by 6 months. ${ }^{11}$ We postulate that the benefit that the addition of ipilimumab confers to tumor shrinkage outweighs the possible increased inflammatory milieu, resulting in an overall reduction in edema. In addition, at 3 and 6 months after SRS, concurrent ipilimumab was associated with the highest rate of lesion response and the lowest rate of lesion progression, supporting 2 recent studies that found that ipilimumab administered concurrently with SRS was associated with greater tumor volume reduction and improved locoregional control. ${ }^{8,18}$

Finally, we found that SRS dose $\geq 20$ Gy was associated with significantly greater median tumor volume reduction at 3 and 6 months within the concurrent ipilimumab group, a relationship that was not present in the nonconcurrent ipilimumab and control groups. This finding suggests that there may be a radiation dose-response relationship when SRS and ipilimumab are administered concurrently.

\section{Local Failure}

While the crude incidence of local failure was similar in all groups, on univariate survival analysis we found that concurrent ipilimumab was associated with lower risk of local failure. In multivariate analysis, due to the fact that tumor and edema response were related to ipilimumab ad- 
TABLE 5. Univariate and multivariate analyses of selected outcomes

\begin{tabular}{|c|c|c|c|c|}
\hline \multirow[b]{2}{*}{ Variable } & \multicolumn{2}{|l|}{ Univariate } & \multicolumn{2}{|l|}{ Multivariate } \\
\hline & $\mathrm{HR}(95 \% \mathrm{Cl})$ & $p$ Value & $\mathrm{HR}(95 \% \mathrm{Cl})$ & $p$ Value \\
\hline \multicolumn{5}{|l|}{ Local failure } \\
\hline \multicolumn{5}{|l|}{ Treatment group } \\
\hline No ipilimumab & Ref & Ref & Ref & Ref \\
\hline Concurrent ipilimumab & $0.342(0.123-0.828)$ & 0.02 & $0.360(0.129-0.873)$ & 0.02 \\
\hline Nonconcurrent ipilimumab & $0.846(0.471-1.569)$ & 0.59 & $0.861(0.478-1.600)$ & 0.63 \\
\hline \multicolumn{5}{|l|}{ Ipilimumab dose } \\
\hline $3 \mathrm{mg} / \mathrm{kg}$ & Ref & Ref & - & - \\
\hline $10 \mathrm{mg} / \mathrm{kg}$ & $1.795(0.793-3.703)$ & 0.15 & - & - \\
\hline \multicolumn{5}{|l|}{ Tumor vol } \\
\hline$<1 \mathrm{~cm}^{3}$ & Ref & Ref & Ref & Ref \\
\hline$>1 \mathrm{~cm}^{3}$ & $2.098(1.077-3.826)$ & 0.03 & $2.020(1.034-3.695)$ & 0.04 \\
\hline \multicolumn{5}{|l|}{ Edema vol } \\
\hline$<2.5 \mathrm{~cm}^{3}$ & Ref & Ref & - & - \\
\hline$>2.5 \mathrm{~cm}^{3}$ & $1.401(0.733-2.525)$ & 0.29 & - & - \\
\hline \multicolumn{5}{|l|}{ Edema index } \\
\hline$<10$ & Ref & Ref & - & - \\
\hline$>10$ & $0.855(0.208-2.330)$ & 0.79 & - & - \\
\hline \multicolumn{5}{|l|}{ Lesion location } \\
\hline Cerebral hemispheres & $1.015(0.444-2.926)$ & 0.98 & - & - \\
\hline Other & Ref & Ref & - & - \\
\hline SRS dose, Gy & $1.11(0.872-1.455)$ & 0.42 & - & - \\
\hline Tumor response at $1.5 \mathrm{mos}$ & $0.234(0.114-0.457)$ & $<0.001$ & $0.498(0.220-1.082)$ & $0.08^{*}$ \\
\hline Edema response at $1.5 \mathrm{mos}$ & $0.300(0.145-0.584)$ & $<0.001$ & $0.522(0.242-1.076)$ & $0.08^{*}$ \\
\hline Tumor response at 3 mos & $0.086(0.034-0.193)$ & $<0.001$ & $0.131(0.047-0.335)$ & $<0.001^{*}$ \\
\hline Edema response at 3 mos & $0.088(0.032-0.204)$ & $<0.001$ & $0.125(0.044-0.309)$ & $<0.001^{*}$ \\
\hline \multicolumn{5}{|l|}{ Any lesion hemorrhage } \\
\hline \multicolumn{5}{|l|}{ Treatment group } \\
\hline No ipilimumab & $0.570(0.274-1.150)$ & 0.12 & $0.469(0.212-1.013)$ & 0.05 \\
\hline Concurrent ipilimumab & Ref & Ref & Ref & Ref \\
\hline Nonconcurrent ipilimumab & $0.480(0.264-0.888)$ & 0.02 & $0.395(0.208-0.763)$ & 0.006 \\
\hline \multicolumn{5}{|l|}{ Ipilimumab dose } \\
\hline $3 \mathrm{mg} / \mathrm{kg}$ & Ref & Ref & - & - \\
\hline $10 \mathrm{mg} / \mathrm{kg}$ & $0.917(0.345-2.046)$ & 0.85 & - & - \\
\hline \multicolumn{5}{|l|}{ Tumor vol } \\
\hline$<1 \mathrm{~cm}^{3}$ & Ref & Ref & - & - \\
\hline$>1 \mathrm{~cm}^{3}$ & $1.528(0.727-2.906)$ & 0.25 & - & - \\
\hline \multicolumn{5}{|l|}{ Edema vol } \\
\hline$<2.5 \mathrm{~cm}^{3}$ & Ref & Ref & - & - \\
\hline$>2.5 \mathrm{~cm}^{3}$ & $0.849(0.404-1.611)$ & 0.63 & - & - \\
\hline \multicolumn{5}{|l|}{ Edema index } \\
\hline$<10$ & Ref & Ref & - & - \\
\hline$>10$ & $1.388(0.482-3.158)$ & 0.50 & - & - \\
\hline \multicolumn{5}{|l|}{ Lesion location } \\
\hline Cerebral hemispheres & $6.868(1.512-121.405)$ & 0.007 & $6.307(1.376-111.784)$ & 0.01 \\
\hline Other & Ref & Ref & Ref & Ref \\
\hline SRS dose, Gy & $1.095(0.876-1.423)$ & 0.45 & - & - \\
\hline Tumor response at $1.5 \mathrm{mos}$ & $0.558(0.318-0.985)$ & 0.04 & $1.063(0.550-2.076)$ & 0.86 \\
\hline Edema response at $1.5 \mathrm{mos}$ & $0.635(0.362-1.118)$ & 0.11 & $1.093(0.577-2.089)$ & 0.79 \\
\hline
\end{tabular}


» CONTINUED FROM PAGE 1403

TABLE 5. Univariate and multivariate analyses of selected outcomes

\begin{tabular}{|c|c|c|c|c|}
\hline \multirow[b]{2}{*}{ Variable } & \multicolumn{2}{|c|}{ Univariate } & \multicolumn{2}{|c|}{ Multivariate } \\
\hline & $\mathrm{HR}(95 \% \mathrm{Cl})$ & p Value & $\mathrm{HR}(95 \% \mathrm{Cl})$ & $\mathrm{p}$ Value \\
\hline \multicolumn{5}{|c|}{ Any lesion hemorrhage (continued) } \\
\hline Tumor response at 3 mos & $0.292(0.158-0.544)$ & $<0.001$ & $0.225(0.107-0.476)$ & $<0.001$ \\
\hline Edema response at 3 mos & $0.301(0.162-0.555)$ & $<0.001$ & $0.262(0.130-0.523)$ & $<0.001$ \\
\hline \multicolumn{5}{|l|}{ Any TRICs } \\
\hline \multicolumn{5}{|l|}{ Treatment group } \\
\hline No ipilimumab & Ref & Ref & - & - \\
\hline Concurrent ipilimumab & $1.283(0.457-3.681)$ & 0.63 & - & - \\
\hline Nonconcurrent ipilimumab & $1.145(0.490-2.980)$ & 0.76 & - & - \\
\hline \multicolumn{5}{|l|}{ Ipilimumab dose } \\
\hline $3 \mathrm{mg} / \mathrm{kg}$ & Ref & Ref & - & - \\
\hline $10 \mathrm{mg} / \mathrm{kg}$ & $0.687(0.162-2.011)$ & 0.53 & - & - \\
\hline \multicolumn{5}{|l|}{ Tumor vol } \\
\hline$<1 \mathrm{~cm}^{3}$ & Ref & Ref & - & - \\
\hline$>1 \mathrm{~cm}^{3}$ & $1.160(0.392-2.773)$ & 0.77 & - & - \\
\hline \multicolumn{5}{|l|}{ Edema vol } \\
\hline$<2.5 \mathrm{~cm}^{3}$ & Ref & Ref & - & - \\
\hline$>2.5 \mathrm{~cm}^{3}$ & $1.065(0.425-2.335)$ & 0.88 & - & - \\
\hline \multicolumn{5}{|l|}{ Edema index } \\
\hline$<10$ & Ref & Ref & - & - \\
\hline$>10$ & $0.418(0.023-1.947)$ & 0.32 & - & - \\
\hline \multicolumn{5}{|l|}{ Lesion location } \\
\hline Cerebral hemispheres & $3.767(0.810-67.015)$ & 0.10 & - & - \\
\hline Other & Ref & Ref & - & - \\
\hline SRS dose, Gy & $0.933(0.728-1.258)$ & 0.62 & - & - \\
\hline Tumor response at 1.5 mos & $0.138(0.055-0.308)$ & $<0.001$ & $0.144(0.053-0.359)$ & $<0.001$ \\
\hline Edema response at $1.5 \mathrm{mos}$ & $0.219(0.091-0.476)$ & $<0.001$ & $0.297(0.116-0.703)$ & 0.005 \\
\hline Tumor response at 3 mos & $0.298(0.131-0.675)$ & 0.004 & $0.867(0.346-2.120)$ & 0.76 \\
\hline Edema response at 3 mos & $0.248(0.106-0.558)$ & $<0.001$ & $0.443(0.176-1.066)$ & 0.07 \\
\hline
\end{tabular}

* Due to collinearity between model terms, these risk factors were analyzed separately.

ministration and baseline tumor volume (variance inflation factor [VIF] of 20.8, where VIF > 10 typically represents collinearity), we performed a separate analysis for early tumor and edema response. ${ }^{26}$ After adjusting for tumor volume, concurrent ipilimumab remained associated with lower risk for local failure. Tumor and edema response at 3 months were ultimately the most powerful predictors of local control, consistent with a study by Sharpton et al.,20 who found that tumor volume reduction at 6 and 12 weeks was associated with prolonged local control.

\section{Lesion Hemorrhage}

There was greater incidence of lesion hemorrhage within the concurrent ipilimumab group, but this difference did not translate to a higher incidence of symptomatic lesion hemorrhage. In univariate analysis, we found that nonconcurrent ipilimumab was associated with a lower risk of lesion hemorrhage compared with concurrent ipilimumab. Tumor and edema response at 3 months were also associated with lower risk for lesion hemorrhage. A cerebral hemisphere location of tumor was associated with a higher risk of lesion hemorrhage. In multivariate analysis, all of these risk factors remained significant. We hypothesize that the observed increased risk of lesion hemorrhage in cerebral hemisphere location tumors could be due to increased vascularity of brain tissue in the cerebral cortex., ${ }^{416}$ Previous studies have not found any association of ipilimumab administration with lesion hemorrhage; however, these were primarily retrospective studies with relatively small sample sizes and may not have accounted for mild or asymptomatic lesion hemorrhages detected on imaging. ${ }^{14,22}$ Taken together, our results suggest that concurrent ipilimumab is associated with a greater risk for subclinical lesion hemorrhage.

\section{TRICs and RN}

The overall incidence of TRICs was $10 \%$ and was similar across the groups. Patients who received ipilimumab, however, had significantly higher rates of symptomatic TRICs, with $8 \%$ and $6 \%$ developing symptomatic TRICs 
in the concurrent and nonconcurrent ipilimumab groups, respectively, compared with $0 \%$ in the control group. In univariate analysis of any TRICs, only tumor and edema response at 1.5 and 3 months were protective for TRICs. In multivariate analysis, tumor and edema response at 1.5 months were the strongest protective factors for any TRICs. Among lesions that developed into TRICs, the concurrent ipilimumab group had the largest TRICs, followed by nonconcurrent ipilimumab and no ipilimumab, although the difference was not significant. These findings suggest that whether TRICs were symptomatic or not was likely related to the size of the TRICs. We postulate that the higher incidence of symptomatic TRICs in patients receiving ipilimumab could be due to either less treatment of TRICs with steroids because of concerns that steroids may lessen the efficacy of ipilimumab, or a proinflammatory effect of ipilimumab that occurs only in select patients. ${ }^{8,24}$

Due to the low incidence of pathologically confirmed $\mathrm{RN}$ in our cohort, we did not report univariate or multivariate analysis findings in our results. However, the low incidence of RN in the ipilimumab groups supports the relative safety of combination treatment. Other studies have found rates of RN after SRS ranging from $2 \%$ to more than $30 \%$ owing to heterogeneous cohorts and the difficulty in accurately defining and diagnosing RN. ${ }^{10}$ Colaco et al. $^{3}$ found that patients receiving SRS and immunotherapy had higher rates of RN/TRICs compared with patients receiving SRS and chemotherapy or targeted therapy. We now report that patients receiving SRS and ipilimumab may be at higher risk for symptomatic TRICs.

\section{Limitations}

Limitations of the present study include its retrospective nature, the lack of pathology on the majority of followed lesions to differentiate RN from pseudoprogression and tumor recurrence, the lack of data on steroid administration to account for changes in tumor and edema volumes, and some imprecision in distinguishing lesion hemorrhage from melanotic metastases. As this was primarily a radiographic imaging-based analysis, our outcomes may not be clinically applicable in all situations.

\section{Conclusions}

To our knowledge, this is the first study to examine edema trajectory following brain metastasis treatment with SRS and immunotherapy. Edema trajectory closely mirrored that of tumor trajectory. Patients receiving ipilimumab had greater tumor and edema volume reduction, with concurrent ipilimumab demonstrating the highest rates of tumor response, lowest rates of tumor progression, and less risk for local failure. SRS dose $\geq 20$ Gy was associated with greater median tumor volume reduction in the concurrent ipilimumab group, suggesting a radiation dose-response relationship between SRS and ipilimumab when administered concurrently. Early tumor and edema response were excellent predictors of local failure, lesion hemorrhage, and TRICs. Concurrent ipilimumab was associated with increased risk for lesion hemorrhage overall but not symptomatic lesion hemorrhage. Although any ipilimumab was associated with higher incidence of symp- tomatic TRICs, the incidence of pathologically proven RN in lesions receiving any ipilimumab was $2 \%$, supporting the relative safety of ipilimumab in SRS treatment.

\section{Acknowledgments}

We wish to gratefully acknowledge the research support of the Kozak, Ginsburg, and Phillip families for the research stipend of Kevin Diao. Our sponsors had no involvement in the research project.

\section{References}

1. Brown PD, Jaeckle K, Ballman KV, Farace E, Cerhan JH, Anderson SK, et al: Effect of radiosurgery alone vs radiosurgery with whole brain radiation therapy on cognitive function in patients with 1 to 3 brain metastases: a randomized clinical trial. JAMA 316:401-409, 2016

2. Chang EL, Wefel JS, Hess KR, Allen PK, Lang FF, Kornguth DG, et al: Neurocognition in patients with brain metastases treated with radiosurgery or radiosurgery plus whole-brain irradiation: a randomised controlled trial. Lancet Oncol 10:1037-1044, 2009

3. Colaco RJ, Martin P, Kluger HM, Yu JB, Chiang VL: Does immunotherapy increase the rate of radiation necrosis after radiosurgical treatment of brain metastases? J Neurosurg 125:17-23, 2016

4. Dunning HS, Wolff HG: The relative vascularity of various parts of the central and peripheral nervous system of the cat and its relation to function. J Comp Neurol 67:433-450, 1937

5. Fellner C: Ipilimumab (yervoy) prolongs survival in advanced melanoma: serious side effects and a hefty price tag may limit its use. P\&T 37:503-530, 2012

6. Gebel JM, Sila CA, Sloan MA, Granger CB, Weisenberger JP, Green CL, et al: Comparison of the $\mathrm{ABC} / 2$ estimation technique to computer-assisted volumetric analysis of intraparenchymal and subdural hematomas complicating the GUSTO-1 trial. Stroke 29:1799-1801, 1998

7. Hodi FS, O'Day SJ, McDermott DF, Weber RW, Sosman JA, Haanen JB, et al: Improved survival with ipilimumab in patients with metastatic melanoma. N Engl J Med 363:711723, 2010

8. Kiess AP, Wolchok JD, Barker CA, Postow MA, Tabar V, Huse JT, et al: Stereotactic radiosurgery for melanoma brain metastases in patients receiving ipilimumab: safety profile and efficacy of combined treatment. Int J Radiat Oncol Biol Phys 92:368-375, 2015

9. Knisely JP, Yu JB, Flanigan J, Sznol M, Kluger HM, Chiang VL: Radiosurgery for melanoma brain metastases in the ipilimumab era and the possibility of longer survival. J Neurosurg 117:227-233, 2012

10. Kohutek ZA, Yamada Y, Chan TA, Brennan CW, Tabar V, Gutin PH, et al: Long-term risk of radionecrosis and imaging changes after stereotactic radiosurgery for brain metastases. J Neurooncol 125:149-156, 2015

11. Konstantinou MP, Dutriaux C, Gaudy-Marqueste C, Mortier L, Bedane C, Girard C, et al: Ipilimumab in melanoma patients with brain metastasis: a retro-spective multicentre evaluation of thirty-eight patients. Acta Derm Venereol 94:45-49, 2014

12. Kothari RU, Brott T, Broderick JP, Barsan WG, Sauerbeck LR, Zuccarello M, et al: The ABCs of measuring intracerebral hemorrhage volumes. Stroke 27:1304-1305, 1996

13. Kroeze SGC, Fritz C, Hoyer M, Lo SS, Ricardi U, Sahgal A, et al: Toxicity of concurrent stereotactic radiotherapy and targeted therapy or immunotherapy: a systematic review. Cancer Treat Rev 53:25-37, 2017

14. Mathew M, Tam M, Ott PA, Pavlick AC, Rush SC, Donahue 
$\mathrm{BR}$, et al: Ipilimumab in melanoma with limited brain metastases treated with stereotactic radiosurgery. Melanoma Res 23:191-195, 2013

15. Nabors LB, Ammirati M, Bierman PJ, Brem H, Butowski N, Chamberlain MC, et al: Central nervous system cancers. J Natl Compr Canc Netw 11:1114-1151, 2013

16. Nair V, Palm D, Roth LJ: Relative vascularity of certain anatomical areas of the brain and other organs of the rat. Nature 188:497-498, 1960

17. Pan HC, Sun MH, Chen CCC, Chen CJ, Lee CH, Sheehan $\mathrm{J}$ : Neuroimaging and quality-of-life outcomes in patients with brain metastasis and peritumoral edema who undergo Gamma Knife surgery. J Neurosurg 109 Suppl:90-98, 2008

18. Qian JM, Yu JB, Kluger HM, Chiang VLS: Timing and type of immune checkpoint therapy affect the early radiographic response of melanoma brain metastases to stereotactic radiosurgery. Cancer 122:3051-3058, 2016

19. Robert C, Thomas L, Bondarenko I, O'Day S, Weber J, Garbe C, et al: Ipilimumab plus dacarbazine for previously untreated metastatic melanoma. N Engl J Med 364:25172526, 2011

20. Sharpton SR, Oermann EK, Moore DT, Schreiber E, Hoffman R, Morris DE, et al: The volumetric response of brain metastases after stereotactic radiosurgery and its post-treatment implications. Neurosurgery 74:9-16, 2014

21. Shaw E, Scott C, Souhami L, Dinapoli R, Kline R, Loeffler J, et al: Single dose radiosurgical treatment of recurrent previously irradiated primary brain tumors and brain metastases: final report of RTOG protocol 90-05. Int J Radiat Oncol Biol Phys 47:291-298, 2000

22. Silk AW, Bassetti MF, West BT, Tsien CI, Lao CD: Ipilimumab and radiation therapy for melanoma brain metastases. Cancer Med 2:899-906, 2013

23. Sneed PK, Mendez J, Vemer-van den Hoek JGM, Seymour ZA, Ma L, Molinaro AM, et al: Adverse radiation effect after stereotactic radiosurgery for brain metastases: incidence, time course, and risk factors. J Neurosurg 123:373-386, 2015
24. Tarhini A: Immune-mediated adverse events associated with ipilimumab CTLA-4 blockade therapy: the underlying mechanisms and clinical management. Scientifica (Cairo) 2013:857519, 2013

25. Tazi K, Hathaway A, Chiuzan C, Shirai K: Survival of melanoma patients with brain metastases treated with ipilimumab and stereotactic radiosurgery. Cancer Med 4:1-6, 2015

26. Tu YK, Kellett M, Clerehugh V, Gilthorpe MS: Problems of correlations between explanatory variables in multiple regression analyses in the dental literature. Br Dent $\mathbf{J}$ 199:457-461, 2005

27. Wolchok JD, Hoos A, O’Day S, Weber JS, Hamid O, Lebbé $\mathrm{C}$, et al: Guidelines for the evaluation of immune therapy activity in solid tumors: immune-related response criteria. Clin Cancer Res 15:7412-7420, 2009

\section{Disclosures}

Eric L. Chang reports receipt of a speaker's honorarium from Brainlab.

\section{Author Contributions}

Conception and design: Diao, Chang. Acquisition of data: Diao. Analysis and interpretation of data: Diao, Bian, Routman, Chang. Drafting the article: Diao, Bian, Routman, Chang. Critically revising the article: all authors. Reviewed submitted version of manuscript: all authors. Approved the final version of the manuscript on behalf of all authors: Diao. Statistical analysis: Diao. Administrative/technical/material support: Yu, Kim, Chang. Study supervision: Wagle, Wong, Zada, Chang.

\section{Correspondence}

Kevin Diao: Harvard Medical School, Boston, MA. kevin_diao@ hms.harvard.edu. 